\title{
Choose Your Country to Preserve Your Quality of Life
}

\author{
Raffaele Antonelli-Incalzi ${ }^{a} \quad$ Nicola Scichilone ${ }^{b}$

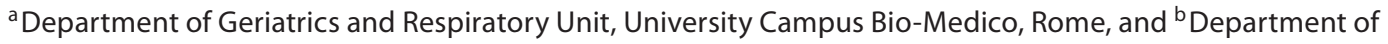 \\ Internal Medicine, Division of Pulmonology, University of Palermo, Villa Sofia-Cervello Hospital, Palermo, Italy
}

Health-related quality of life (HRQoL) is gaining increasing popularity as an index of disease severity and an important predictor of survival in chronic respiratory diseases, being more and more frequently employed as an outcome of intervention trials in respiratory medicine. HRQoL has important classificatory and predictive properties and is highly sensible to comorbid conditions and sociodemographic factors, thus being much more comprehensive than traditional indexes of respiratory function. Furthermore, it is measurable even in patients with the most severe forms of chronic obstructive pulmonary disease (COPD), who are frequently unable to undergo a satisfactory lung function assessment [1]. Confirming the distinctive role of HRQoL for both clinical and epidemiological purposes is the fact that about one quarter of COPD patients $>64$ years old with a forced expiratory volume in $1 \mathrm{~s}>50 \%$ have the worst HRQoL, while $15 \%$ of those with a forced expiratory volume in $1 \mathrm{~s}<35 \%$ score in the highest HRQoL tertile [2]. Thus, HRQoL qualifies as a valuable measure to help the respiratory physician in the diagnostic process and monitoring of the disease, provided that she/he has sufficient insight into the meaning and the determinants of HRQoL.

Clinical and sociodemographic factors contributing to explain HRQoL have been the object of several studies. In COPD individuals $>65$ years of age, poor physical performance, depressed mood, polypharmacy and composition of household seem to be the main correlates of a poor HRQoL $[3,4]$. In younger COPD populations, factors that predict HRQoL seem to be gender dependent. In a study by de Torres and colleagues [5], in which COPD subjects as young as 37 years were enrolled, only dyspnea and the level of arterial oxygenation contributed to the score in women $\left(r^{2}=0.47\right)$. In general, women appear to show worse HRQoL scores than men, perhaps because of the contribution of psychological impairment. Interestingly, the relation between emotional distress and HRQoL in younger subjects is interactive within a couple, meaning that the spouses' well-being (i.e. anxiety and depression) significantly impacts the HRQoL [6].

To this bulk of evidence, in this issue of Respiration, Fan and co-authors [7] add the important observation that regional differences may act as a major source of variability in HRQoL. Indeed, the authors found that regional differences were greater for the physical dimension of HRQoL irrespective of whether it is rated by a generic index, the Medical Outcomes Study 36-item Short Form (SF-36), or a disease-specific questionnaire, the Seattle Obstructive Lung Disease Questionnaire (SOLDQ). The largest proportion of variance in HRQoL cumulatively explained by a multifactorial model (site, sociodemographic factors, comorbidity, use of health care resources, amount of prescribed COPD medication, clinical factors) was observed in the neuropsychological domain of SF-36. Most of the variance was explained by comorbidity, especially for the SF-36. However, the total variance in HRQoL cumulatively explained by the best model ranged from 19.1\% (SOLDQ, coping skills) to

\section{KARGER}

Fax +41613061234 E-Mail karger@karger.ch www.karger.com
(C) 2010 S. Karger AG, Basel 0025-7931/11/0811-0004\$38.00/0

Accessible online at: www.karger.com/res
Nicola Scichilone

Istituto di Medicina Generale e di Pneumologia dell'Università di Palermo

Via Trabucco, 180

IT-90146 Palermo (Italy)

Tel. +39 091680 2654, Fax +39 091688 2165, E-Mail n.scichilone@libero.it 
29.3\% (SF-36, mental component summary). Analogously, in the Spanish EPIDEPOC multicenter study, the physical and mental components of the SF-12 questionnaire, the short form of the SF-36, explained a variance of 31 and $11 \%$, respectively [8]. It is worthy observing that even a simpler purely 'physical' index such as the 6-min walking test has about $60 \%$ variance unexplained even if the explanatory model has been developed in a healthy, and thus, relatively homogeneous population [9]. Thus, the up to $30 \%$ explained variance in HRQoL in the study by Fan et al. [7] should be considered a fair result, although it might partly reflect selected limitations, which the authors properly acknowledge. More important, HRQoL is primarily a subjective dimension and, as such, difficult to explain on purely mechanistic bases. Even a consistent proportion of paraplegic patients, almost completely dependent on external help, have been reported to rate their HRQoL as satisfactory [10].

Related to the responsiveness criterion of a HRQoL questionnaire is the threshold for a clinically meaningful change, which might vary in relation to even geographical differences. Thus, any attempt at improving our understanding of the interpretable variance in HRQoL is welcome. From this perspective, becoming aware that geographical differences may account for about a quarter or a fifth of the cumulatively explained variance in the physical dimension of both a disease-specific and a generic HRQoL questionnaire substantially adds to our culture and has potentially important implications. First, regional differences in HRQoL should be carefully taken into account by health care decision makers as a clue to modifiable conditions affecting health status, by properly managing health resources. The cost-effectiveness of COPD interventions are currently assessed in many European and North American countries including outcomes related to QoL. Second, baseline geographical heterogeneity in HRQoL might herald differences in response to intervention trials, and this might occur through unobvious mechanisms. For instance, patients with depressed HRQoL are more likely to lead a sedentary life and to adhere less to prescribed therapy, both factors being usually unmeasured, and thus, affecting the results of any intervention trial. It follows that subgroup analysis, which usually completes the core analysis of a trial, should also take into account inter-site variability besides the usual dimensions of researchers' performance or severity of obstruction, especially if, as it frequently occurs, the individual centers largely differ in the proportions of enrolled patients.

The authors should be commended for having shed light on a potentially important issue. Further research is needed to gain insight into determinants of inter-site variability of HRQoL. If present results will be confirmed by a more complete interpretative model, both health authorities and trialists should become aware of their implications. Indeed, both planning health/social interventions and designing/interpreting multicenter trials will benefit from a thorough knowledge of regional differences in HRQoL and related explanatory mechanisms. Eventually, what the patient asks for is not how much she/he improved her/his lung function, rather how well she/he feels!

\section{References}

1 Bellia V, Pistelli R, Catalano F, AntonelliIncalzi R, Grassi V, Melillo G, Olivieri D, Rengo F: Quality control of spirometry in the elderly. The SA.R.A. study. SAlute Respiration nell'Anziano = Respiratory Health in the Elderly. Am J Respir Crit Care Med 2000; 161:1094-1100.

2 Antonelli-Incalzi R, Pedone C, Pahor M: Multidimensional Assessment and Treatment of the Elderly with COPD. ERS Monogr No 43. European Respiratory Society, 2009, pp 35-55.

3 Antonelli Incalzi R, Bellia V, Catalano F, Scichilone N, Imperiale C, Maggi S, Rengo F: Evaluation of health outcomes in elderly patients with asthma and COPD using diseasespecific and generic instruments: the Salute Respiratoria nell'Anziano (Sa.R.A.) Study. Chest 2001;120:734-742.
-4 Incalzi RA, Bellia V, Maggi S, Imperiale C, Capparella O, Pistelli R, Grassi V, Sa.R.A. Study Group: Reversible bronchial obstruction and disease-related health status in COPD. Qual Life Res 2002;11:517-525.

$\checkmark 5$ de Torres JP, Casanova C, Hernández C, Abreu J, Montejo de Garcini A, Aguirre-Jaime A, Celli BR: Gender associated differences in determinants of quality of life in patients with COPD: a case series study. Health Qual Life Outcomes 2006;4:72.

6 Kühl K, Schürmann W, Rief W: Mental disorders and quality of life in COPD patients and their spouses. Int J Chron Obstruct Pulmon Dis 2008;3:727-736.

7 Fan VS, Bridevaux P-O, McDonell MB, Fihn $\mathrm{SD}$, Besser LM, Au DH: Regional variation in health status among chronic obstructive pulmonary disease patients. Respiration 2011;81:9-17.
-8 Carrasco Garrido P, de Miguel Díez J, Rejas Gutiérrez J, Centeno AM, Gobartt Vázquez E, Gil de Miguel A, García Carballo M, Jiménez García R: Negative impact of chronic obstructive pulmonary disease on the healthrelated quality of life of patients. Results of the EPIDEPOC study. Health Qual Life Outcomes 2006;4:31.

-9 Enright PL, Sherrill DL: Reference equations for the six-minute walk in healthy adults. Am J Respir Crit Care Med 1998;158:13841387.

10 Albrecht GL, Devlieger PJ: The disability paradox: high quality of life against all odds. Soc Sci Med 1998;48:977-988. 